\title{
Object Integrated Recognition of X Band Ground-based Multi-function Radar Based on GRG
}

\author{
LIU Shihua ${ }^{1, a}$ ZHANG Lei $^{2, a}$ \\ ${ }^{1}$ Dept.2, AFEWA, Wuhan 430019, China \\ ${ }^{2}$ Unit 94878, wuhu 241000, China \\ akyoliu2010@sina.com
}

\begin{abstract}
Keywords: object integrated recognition, $\mathrm{X}$ band, multi-function radar, gray relation grade(GRG), effectiveness evaluation

Abstract. According to the integrated recognition problem of the object recognition in the $\mathrm{X}$ band ground-based multi-function radar, an object integrated recognition scheme based on GRG is presented. In this method, the multi-factor statistics and analysis method in the radar effectiveness evaluation. This method is a simply algorithm, it has high credibility and real-time characteristic. The effectiveness of this method is confirmed by the simulation and analysis.
\end{abstract}

\section{Introduction}

The $\mathrm{X}$ band ground-based multi-function radar is a backbone equipment in the antimissile early-warning system. It has multi-function with scan, interception, track, object recognition and battle damage assessment ${ }^{[1-3]}$. The object recognition, which directly affects the antimissile ability of the radar, is a very important function.

The object recognition of the $\mathrm{X}$ band ground-based multi-function radar include three steps ${ }^{[4]}$. Firstly, characteristic extraction, extracting the characteristics of the object. Secondly, object classification, the object is pre-classification by the single characteristic, the confidence level is computed. Finally, integrated recognition, the object is recognized based on all single characteristic pre-classification results, the object class is confirmed.

At present, the method of the integrated recognition in the $\mathrm{X}$ band ground-based multi-function radar is chose to DS evidence theory ${ }^{[5,6]}$ to integrated recognize all pre-classification results. This method is simple, easy to implement. But, the computation amount and time are increased when the object class is added. This is not satisfied the demand of the real-tine recognition. Therefore, an object integrated recognition method of the $\mathrm{X}$ band ground-based multi-function radar is presented based on GRG. This method is solved the real-time problem effectively.

\section{Application of GRG in Object Integrated Recognition of The X Band Ground-Based Multi-Function Radar}

The gray relation grade(GRG) analysis is a multi-factor statistics and analysis method. It has the characteristics of low data demand, sufficient information application, data no distortion. This method is mainly used in radar effectiveness evaluation and other relative domains in order to apply the data integrated. The computation steps are shown as the following ${ }^{[7]}$ :

(1) The individual index weight of $n$ experts for constructed index system are computed by AHP.

(2) Considering that the individual expert weight is unknown, so let the expert weight is equal to $1 / n$. The individual index weight of each expert $\mathbf{W}^{k}(k=1,2, \mathrm{~L}, n)$ is aggregated to the group index weight through weighted method, that is 


$$
\mathbf{W}=\left(w_{1}, w_{2}, \mathrm{~L}, w_{m}\right)^{\mathrm{T}}=\frac{1}{n} \sum_{k=1}^{n} \mathbf{W}^{k}
$$

(3) Because the judgement matrixs of all experts are different, thus, the credibility of individual index weight and group index weight of all experts are disparate. In this paper, the similarity between individual index weight of the $k$-th expert and group index weight is scaled using grey relational grade $r^{k}$, consequently, the expert weight is determined.

The relational coefficient of the $i$-th index between individual index weight obtained by jungement matrix of the $k$-th expert and group index weight is

$$
\xi_{i}^{k}=\frac{\Delta \min +\rho \Delta \max }{\left|w_{i}^{k}-w_{i}\right|+\rho \Delta \max }
$$

Where $\Delta \min =\min _{i} \min _{k}\left|w_{i}^{k}-w_{i}\right|, \Delta \max =\max _{i} \max _{k}\left|w_{i}^{k}-w_{i}\right|, \quad \rho \quad$ is the resolution coefficient and $\rho \in(0,1]$. In order to enhance the resolution, let $\rho=0.1$.

The grey relational grade between individual index weight of the $k$-th expert $\mathbf{W}^{k}$ and group index weight $\mathbf{W}$ is

$$
r^{k}=\frac{1}{m} \sum_{i=1}^{m} \xi_{i}^{k}
$$

Where $r^{k} \leq 1$. The above grey relational grade is normalized and the expert weight vector $\left(\varphi^{1}, \varphi^{2}, \mathrm{~L}, \varphi^{n}\right)$ is determined. The $k$-th expert weight is

$$
\varphi^{k}=\frac{r^{k}}{\sum_{k=1}^{n} r^{k}}
$$

The individual index weight is aggregated based on expert weight of each expert, the new group index weight $\mathbf{W}^{\prime}$ is

$$
\mathbf{W}^{\prime}=\left[\begin{array}{c}
w_{1}^{\prime} \\
w_{2}^{\prime} \\
\mathrm{M} \\
w_{m}^{\prime}
\end{array}\right]=\varphi^{1}\left[\begin{array}{c}
w_{1}^{1} \\
w_{2}^{1} \\
\mathrm{M} \\
w_{m}^{1}
\end{array}\right]+\varphi^{2}\left[\begin{array}{c}
w_{1}^{2} \\
w_{2}^{2} \\
\mathrm{M} \\
w_{m}^{2}
\end{array}\right]+\mathrm{L}+\varphi^{n}\left[\begin{array}{c}
w_{1}^{n} \\
w_{2}^{n} \\
\mathrm{M} \\
w_{m}^{n}
\end{array}\right]
$$

The difference between $\mathbf{W}^{\prime}$ and $\mathbf{W}$ is computed through a range quantization index $d$, that is

$$
d=\sqrt{\sum_{i=1}^{m}\left(w_{i}-w_{i}^{\prime}\right)^{2}}
$$

The threshold $d_{0}$ is set according to the precision requirement of evaluation. When $d \leq d_{0}$, it means the difference between $\mathbf{W}^{\prime}$ and $\mathbf{W}$ is very small and tend to stability, this new group index weight $\mathbf{W}^{\prime}$ is index weight of the layer of concern. Otherwise, the $\mathbf{W}$ is replaced by new group index weight $\mathbf{W}^{\prime}$, the grey relational grade between each index weight and new group 
index weight is computed by "Eq.(2)" and "Eq.(3)", and the expert weight vector $\left(\varphi^{1}, \varphi^{2}, \mathrm{~L}, \varphi^{n}\right)$ is obtained. The group index weight $\mathbf{W}^{\prime}$ is renewed by substituting expert weight vector into "Eq.(5)". Then, compute the difference $d$ between $\mathbf{W}^{\prime}$ and $\mathbf{W}$. Repeat the above steps until $d \leq d_{0}$.

In the above algorithm, let expert is characteristic, let individual index weight is confidence level computation result based on the single characteristic, let group index weight is integrated recognition result, the object integrated recognition based on GRG is implemented.

\section{Simulations and Analysis}

Assume the object recognition frame is $\Theta=\left\{h_{1}, h_{2}, h_{3}, h_{4}, h_{5}\right\}$, that is, there are five kinds detection object. The object confidence levels based on the single characteristic classification are shown as table 1 . Where $m_{1}$ is the RCS characteristic, $m_{2}$ is the micro-motion characteristic, $\mathrm{m}_{3}$ is the polarimetric characteristic, $\mathrm{m}_{4}$ is the $1-\mathrm{D}$ profile characteristic, $\mathrm{m}_{5}$ is the posture information.

Table 1 object confidence levels based on the single characteristic classification

\begin{tabular}{|c|c|c|c|c|c|}
\hline & $h_{1}$ & $h_{2}$ & $h_{3}$ & $h_{4}$ & $h_{5}$ \\
\hline$m_{1}$ & 0.15 & 0.35 & 0.08 & 0.24 & 0.18 \\
\hline$m_{2}$ & 0.12 & 0.38 & 0.09 & 0.22 & 0.19 \\
\hline$m_{3}$ & 0.14 & 0.41 & 0.06 & 0.26 & 0.13 \\
\hline$m_{4}$ & 0.16 & 0.37 & 0.10 & 0.21 & 0.16 \\
\hline$m_{5}$ & 0.13 & 0.40 & 0.11 & 0.19 & 0.17 \\
\hline
\end{tabular}

The data is analyzed and computed by GRG method. The confidence level vectors based on the single characteristic classification are $\quad \mathbf{W}^{1}=\left(\begin{array}{lllll}0.15 & 0.35 & 0.08 & 0.24 & 0.18\end{array}\right)^{\mathrm{T}}, \quad \mathbf{W}^{2}=\left(\begin{array}{ll}0.12 & 0.38\end{array}\right.$ $\left.\begin{array}{lll}0.09 & 0.22 & 0.19\end{array}\right)^{\mathrm{T}}, \quad \mathbf{W}^{3}=\left(\begin{array}{lllll}0.14 & 0.41 & 0.06 & 0.26 & 0.13\end{array}\right)^{\mathrm{T}}, \quad \mathbf{W}^{4}=\left(\begin{array}{lllll}0.16 & 0.37 & 0.10 & 0.21\end{array}\right.$ $0.16)^{\mathrm{T}}, \quad \mathbf{W}^{5}=\left(\begin{array}{lllll}0.13 & 0.40 & 0.11 & 0.19 & 0.17\end{array}\right)^{\mathrm{T}}$. The integrated recognition result $\mathbf{W}=(0.140$ $\left.\begin{array}{llll}0.382 & 0.088 & 0.224 & 0.166\end{array}\right)^{\mathrm{T}}$ is obtained by Eq.1. The new integrated recognition confidence level vector $\mathbf{W}^{\prime}=\left(\begin{array}{lllll}0.1374 & 0.3831 & 0.0873 & 0.2246 & 0.1675\end{array}\right) \mathrm{T}$ is achieved by Eq.2 to Eq.5. Here $d=0.003$. In this paper $d_{0}=0.001$, hence, $d>d_{0}$. Let $\mathbf{W}=\mathbf{W}^{\prime}$, the integrated recognition confidence level is adjusted by Eq.2 to Eq.5 to satisfied the demand of the precision.

The final confidence level vector is $\mathbf{W}^{\prime}=\left(\begin{array}{lllll}0.138 & 0.383 & 0.094 & 0.215 & 0.169\end{array}\right)^{\mathrm{T}}$. That means the object is most probably belongs to $h_{2}$. The result show that the integrated recognition method based on GRG have good recognition ability.

\section{Conclusions}

In this paper, the GRG group decision method is analyzed. The GRG group decision theory is 
introduced in the object integrated recognition of the $\mathrm{X}$ band ground-based multi-function radar and the object integrated recognition method is presented based on GRG. In the simulation and analysis, the integrated confidence level is computed and the algorithm feasibility is analyzed. From the computation result we can conclude that this method is fully applied all data information, the data result is believable, the integrated recognition result is pretty good.

\section{References}

[1] J. L. Ren, Q. H. Xing. Research on deployment of X band radar detecting multi-trajectories in early warning system of missile defense[J]. Modern Defence Technology, 2013, 41(6): 20-24.

[2] X. C. Fo, X. R. Jia, Q, S, Lin. Analysis of X-band radar' s capability in missile defense system[J]. Fire Control Radar Technology, 2009, 38(3): 8-12.

[3] X. D. Chen. Development status of US sea-based X-band radar system[J]. Modern Radar, 2011, 33(6): 29-31.

[4] W. X. Zhou. BMD radar target recognition technology[M]. Beijing: Publishing House of Electronics Industry, 2011.

[5] Y. J. Han, G. H. Wang, N. Wang. Multi-radar target identification method based on DS evidence theory[J]. Journal of Projectiles,Rockets,Missiles and Guidance, 2009, 29(5): 215-220.

[6] Q. H. Li, J. C. Li, Z, K, Shen, et al. IR Small Target Recognition Based on the D-S Evidential Theory[J]. Systems Engineering and Electronics, 2002, 24(6): 25-27.

[7] S. H. Liu, W. Sheng, X. H. Zhang. An evaluation scheme of skywave radar coverage of ship target based on GRG[J]. Applied Mechanics and Materials, 2013, 427-429: 888-891. 